\title{
Elastography as a possible useful method of assessment of skin involvement in systemic sclerosis
}

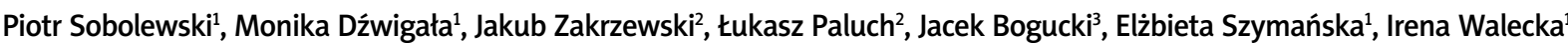 \\ ${ }^{1}$ Clinic of Dermatology, Centre of Postgraduate Medical Education, Central Clinical Hospital of the Ministry of the Interior, Warsaw, \\ Poland \\ ${ }^{2}$ Department of Radiology, Centre of Postgraduate Medical Education, Gruca Orthopaedic and Trauma Teaching Hospital, Otwock, \\ Poland \\ ${ }^{3}$ Department of Organic Chemistry, Faculty of Pharmacy, Medical University, Lublin, Poland
}

Adv Dermatol Allergol 2022; XXXIX (4): 775-781

DOI: https://doi.org/10.5114/ada.2021.109503

\begin{abstract}
Introduction: Scleroderma (Sc) is a connective tissue disorder associated with internal organ involvement, increased mortality, and unknown pathogenesis. It has been found that the more extensive the skin involvement the more severe internal organ manifestations and increased disability. The Rodnan skin score (RSS) is one of the established methods to examine skin thickness among patients with Sc. Due to RSS limitations, for instance, lack of detection of subclinical changes, a new tool is needed for the evaluation of Sc. In recent studies, shear wave elastography (SWE) has been examined as a potential tool to assess skin involvement through the evaluation of skin strain.

Aim: To verify whether elastography is a reliable method to examine Sc progression and possibly provide one useful site to perform the examination - as an easy, cheap, and reliable examination tool.

Material and methods: Forty Sc patients were examined, and 28 healthy individuals were recruited for the control group. Among the patients and control group, skin thickness was assessed using the RSS and skin strain measurements using elastography in 20 body locations.

Results: SWE in the right-hand finger can be treated as an important diagnostic indicator of the severity of Sc. Conclusions: SWE is a reliable method for evaluating skin involvement among patients with systemic sclerosis (SSc). Right finger measurements correlate positively with Rodnan's results and can be a predictor of the severity of SSc. This study found SWE to be a reliable method for examining SSc progression and possibly one useful site for the examination.
\end{abstract}

Key words: sclerosis, skin, thickness, Rodnan skin score, elastography.

\section{Introduction}

Scleroderma (Sc) is a connective tissue disease whose pathogenesis is still unknown. There are two main types of Sc, localized and systemic. Systemic sclerosis (SSc) consists of limited cutaneous systemic sclerosis (previously known as the CREST syndrome) and diffuse cutaneous SSc. Systemic sclerosis is associated with internal organ involvement and increased mortality. The classification of SSc is based on skin involvement [1]. Limited cutaneous systemic sclerosis (LCSSc) is associated with skin thickening distal to the elbows, distal to the knees, and/or face without trunk involvement. Diffuse cutaneous systemic sclerosis (DcSSc) is associated with skin thickening that can involve skin proximal to the elbows, proximal to the knees, face, and/or trunk. Internal organs usually affected by scleroderma are the gastrointestinal tract, lungs, kidneys, skeletal muscle, and pericardium [2-4].

The Rodnan skin score (RSS) is a method of assessing skin involvement among patients with SSc. The RSS is an established method of examining skin thickness in SSc patients. It is a semiquantitative, non-invasive, rapid method to measure skin thickness with high reproducibility [5]. Thus, it is widely used both in clinical trials and clinical practice. Previous studies have investigated the relationship between the incidence of disease-related events or the presence of organ involvement and skin thickness that was quantitatively evaluated [6-10]. Skin thickening is a characteristic feature of SSc. The more

Address for correspondence: Piotr Sobolewski, Clinic of Dermatology, Centre of Postgraduate Medical Education, Central Clinical Hospital of the Ministry of the Interior, Warsaw, Poland, phone: +48 22508 1482, fax: +48 22508 1492, e-mail: piotr.sobolewski@cskmswia.pl Received: 24.05.2021, accepted: 3.08.2021. 
extensive a patient's skin involvement is the more severe the internal organ manifestation(s), the poorer the prognosis, and the greater the disability.

The modified Rodnan Skin Score (mRSS), introduced in 1979, is a common method for assessing the severity of skin involvement [6]. It is a palpation-based, semiquantitative score used in the quantitative estimation of skin hardening measured in 17 areas of the body, contrary to classic RSS (cRSS) in which 20 body areas are checked. Each examined area is assessed on a 4-point scale from 0 - representing no skin hardness, to 3 - representing severe skin thickness (inability to make the skin fold between two fingers). Each area is added together to give an overall result ranging from 0 (no hardening) to 51 (severe hardening of the skin in all 17 areas). The RSS score divides the severity of skin lesions into: mild (1-14), moderate (15-29), severe (30-39), or terminal ( $\geq 40$ ).

The mRSS is an established factor influencing the prognosis due to its accurate reflection of skin biopsy thickness in SSc [6]. Studies have shown that the severity of skin hardening assessed using the mRSS was predictive for disease outcome [7]. The limitations of using the mRSS include examiner's experience and skills as well as difficulties in standardization among different centres [11]. Moreover, the mRSS may not be sensitive enough to detect small but relevant (subclinical) changes in skin thickness over time [12]. Nevertheless, the mRSS is preferred among clinicians as a non-invasive and relatively cheap method [11]. Due to the limitations of the mRSS, an objective and sensitive method of skin assessment in SSC is still under investigation. Several non-invasive methods for quantifying skin involvement in SSc have been described over the past 10 years [11-13]. Ultrasound (US) skin imaging, high-frequency B-mode ultrasonography, ultrasound shear-wave elastography (US-SWE), acoustic radiation force impulse (ARFI) imaging, and magnetic resonance imaging are frequently considered tools for the skin assessment in SSc. However, ultrasound has yet to be confirmed as a reliable method of skin involvement imaging in SSc. It is also required to establish the standard operating procedure of patient examination to make sure that the new tool is quick, repeatable, and easy to learn.

\section{Aim}

This study aims to verify whether elastography is a reliable method to examine SSc progression and possibly provide one useful place to examine to be an easy, cheap, and reliable examination tool.

\section{Material and methods}

The study was conducted between 31 October 2018 and 23 February 2019, at the Clinic of Dermatology, Centre of Postgraduate Medical Education, Central Clinical Hospital of the Ministry of the Interior, Warsaw, Poland.

\section{Study participants}

Forty patients with a confirmed diagnosis of systemic sclerosis based on the ACR and EULAR classification criteria were recruited to participate in the study (either with dSS or ISSc) [14]. Out of the 40 patients with SSc, $34(85.0 \%)$ patients were female (F) and $6(15.0 \%)$ were male (M); F/M ratio was $4: 1$. The age of the patients ranged from 23 to 77 years (mean: $44.4 \pm 13.5$ years). Twenty-nine $(72.5 \%)$ of the patients were diagnosed with ISSC and 11 (27.5\%) with dSSc. The median time since the diagnosis of SSC was 9 years (range: $0-30$ years). The control group consisted of 28 healthy sex- and age-matched individuals, 23 (82.1\%) of them were female and 5 (17.9\%) were male, aged between 22 and 71 years old (mean: 44.9 \pm 14.4 y.o.). All examined SSc patients ( $n=40 ; 100.0 \%)$ and 25 (89.3\%) healthy controls used their right hand as the dominant extremity. In both groups, the co-existence of other autoimmune or skin diseases was used as an exclusion criterion to eliminate the influence of other phenomena on the elastography results. The study protocol was approved by the Institutional Bioethics Committee. All patients and healthy volunteers provided informed consent for participation in the study.

\section{Skin assessment}

The skin thickness in patients with SSc was expressed using the classic RSS based on the palpation of 20 cutaneous sites: face, neck, anterior chest, abdomen, upper and lower back, right and left upper arm, right and left forearm, right and left hand, a finger of the right and left hand, right and left thigh, right and left leg, right and left foot. All the anatomical sites used for the RSS evaluation were also assessed with SWE. Each anatomical site was examined separately, with the individual in a supine or prone position. Ultrasonographic scans were obtained with a Toshiba Aplio i900 ultrasound machine (2019 Malaysia) using a 5-18 MHz transducer. Elastography results (strain) were obtained and expressed as previously described by Sobolewski et al. [15].

\section{Statistical analysis}

In order to analyse the relationships between the studied variables, multiple regression and cluster analysis (k-means method) were used. The level of statistical significance was set at $p<0.05$. All calculations were performed using Statistica version 13.3 software (2017; TIBCO Software Inc.).

\section{Results}

Upon analysis, the existence of 3 clusters of examined body areas emerged within the SSc group and control group. Descriptive statistics and distances from the centre of the cluster are presented in the Tables 1-4 and Figures 1 and 2. 
Table 1. Cluster elements and distances from the centre of the cluster; table for the three separate clusters in the SSc group

\begin{tabular}{|c|c|}
\hline \multirow[t]{2}{*}{ Site } & $\begin{array}{c}\text { Cluster elements number } 1 \text { (highest average } \\
\text { results of elastography) and distances from } \\
\text { the centre of the cluster } \\
\text { Focus has } 6 \text { variables }\end{array}$ \\
\hline & Distance \\
\hline Right hand & 14.01380 \\
\hline Right-hand finger & 20.89485 \\
\hline Left hand & 17.48423 \\
\hline Left-hand finger & 13.44973 \\
\hline Left foot & 17.27856 \\
\hline Right foot & 19.99410 \\
\hline \multirow[t]{2}{*}{ Site } & $\begin{array}{l}\text { Cluster elements number } 2 \text { (lowest } \\
\text { average results of elastography) and } \\
\text { distances from the centre of the cluster } \\
\text { Focus has } 10 \text { variables }\end{array}$ \\
\hline & Distance \\
\hline Face & 9.02470 \\
\hline Neck & 7.77815 \\
\hline Anterior chest & 9.76416 \\
\hline Abdomen & 7.80188 \\
\hline Upper back & 6.22601 \\
\hline Lower back & 9.73076 \\
\hline Right upper arm & 16.32964 \\
\hline Left upper arm & 9.91239 \\
\hline Left thigh & 7.89423 \\
\hline Right thigh & 6.67600 \\
\hline \multirow[t]{2}{*}{ Site } & $\begin{array}{c}\text { Cluster elements number } 3 \text { (average mean } \\
\text { results of elastography) and distances } \\
\text { from the centre of the cluster } \\
\text { Focus has } 4 \text { variables }\end{array}$ \\
\hline & Distance \\
\hline Right forearm & 16.34560 \\
\hline Left forearm & 13.09891 \\
\hline Left leg & 14.82378 \\
\hline Right leg & 16.00793 \\
\hline
\end{tabular}

The highest mean results in the control group were obtained in the right and left foot and leg. The highest mean test results in the study group were obtained in the distal parts of the body: fingers of the right and left hand, right and left hand, and the right and left foot, which is consistent with tests performed by other methods.

The regression analysis showed that the elastography examination in the right-hand finger and right forearm had a statistically significant influence on increased Rodnan scores (carried out by the first researcher). The regression analysis also showed that elastography in the right-hand finger and
Table 2. Cluster elements and distances from the centre of the cluster; table for the three distinguished clusters in the control group

\begin{tabular}{|c|c|}
\hline \multirow[t]{2}{*}{ Site } & $\begin{array}{l}\text { Cluster number } 1 \text { elements (average mean } \\
\text { results of elastography) and distances } \\
\text { from the centre of the cluster. } \\
\text { Focus has } 6 \text { variables }\end{array}$ \\
\hline & Distance \\
\hline Right forearm & 5.714451 \\
\hline Right hand & 3.992964 \\
\hline Right-hand finger & 4.497054 \\
\hline Left forearm & 5.144383 \\
\hline Left hand & 4.650279 \\
\hline Left-hand finger & 5.507839 \\
\hline \multirow[t]{2}{*}{ Site } & $\begin{array}{l}\text { Cluster number } 2 \text { elements (highest } \\
\text { average results of elastography) and } \\
\text { distances from the centre of the cluster } \\
\text { Focus has } 4 \text { variables }\end{array}$ \\
\hline & Distance \\
\hline Left leg & 6.013741 \\
\hline Left foot & 6.259103 \\
\hline Right leg & 7.105860 \\
\hline Right foot & 6.770942 \\
\hline \multirow[t]{2}{*}{ Site } & $\begin{array}{l}\text { Cluster elements number } 3 \text { (lowest } \\
\text { average results of elastography) and } \\
\text { distances from the centre of the cluster. } \\
\text { Focus has } 10 \text { variables }\end{array}$ \\
\hline & Distance \\
\hline Face & 3.672430 \\
\hline Neck & 4.645156 \\
\hline Anterior chest & 6.587325 \\
\hline Abdomen & 4.334200 \\
\hline Upper back & 4.713717 \\
\hline Lower back & 4.029976 \\
\hline Right upper arm & 6.146643 \\
\hline Left upper arm & 4.171013 \\
\hline Left thigh & 3.816266 \\
\hline Right thigh & 3.580678 \\
\hline
\end{tabular}

left hand had a statistically significant influence on increased Rodnan scores (when performed by the second researcher).

\section{Discussion}

During the last decade, the applicability of US testing among patients with SSc has been researched to find a tool for the assessment of skin involvement [15-24]. Several studies have shown that US-SWE can be used to quantitatively characterize the degree of skin involvement among patients with systemic sclerosis [15, 20, 21]. 
Table 3. The regression analysis of the results of elastography examination in different areas of the body in correlation to the results of the Rodnan skin score (researcher no. 1)

\begin{tabular}{|c|c|c|c|c|c|c|}
\hline \multirow[t]{2}{*}{$N=38$} & \multicolumn{6}{|c|}{$\begin{array}{c}R=0.93874006 \\
R^{2}=0.88123290 \\
\text { Corrected } R^{2}=0.74150690 \\
F(20.17)=6.3069, p<0.00017 \\
\text { Standard error of estimation: } 2.5700\end{array}$} \\
\hline & $b^{*}$ & $\begin{array}{c}\text { Standard error } \\
\text { of } b^{*}\end{array}$ & $b$ & $\begin{array}{l}\text { Standard error } \\
\text { of } b\end{array}$ & $t(17)$ & $p$ \\
\hline Constant & & & -5.29949 & 2.451941 & -2.16134 & 0.045227 \\
\hline Face & 0.033851 & 0.165572 & 0.01401 & 0.068515 & 0.20445 & 0.840428 \\
\hline Neck & -0.122866 & 0.212332 & -0.06772 & 0.117037 & -0.57865 & 0.570413 \\
\hline Anterior chest & 0.095145 & 0.222547 & 0.03704 & 0.086634 & 0.42753 & 0.674360 \\
\hline Abdomen & 0.143297 & 0.190518 & 0.08454 & 0.112405 & 0.75214 & 0.462252 \\
\hline Upper back & 0.060373 & 0.132623 & 0.04935 & 0.108418 & 0.45522 & 0.654709 \\
\hline Lower back & -0.080656 & 0.170372 & -0.03497 & 0.073872 & -0.47341 & 0.641943 \\
\hline Right upper arm & 0.044530 & 0.149735 & 0.01250 & 0.042023 & 0.29739 & 0.769771 \\
\hline Right forearm & 0.441376 & 0.181627 & 0.10763 & 0.044288 & 2.43012 & 0.026461 \\
\hline Right hand & -0.242195 & 0.245047 & -0.04782 & 0.048385 & -0.98836 & 0.336834 \\
\hline Right-hand finger & 0.570386 & 0.216266 & 0.09330 & 0.035376 & 2.63743 & 0.017286 \\
\hline Left upper arm & -0.096051 & 0.201625 & -0.04164 & 0.087416 & -0.47639 & 0.639865 \\
\hline Left forearm & -0.264756 & 0.273975 & -0.06845 & 0.070838 & -0.96635 & 0.347417 \\
\hline Left hand & 0.324414 & 0.260706 & 0.06205 & 0.049862 & 1.24437 & 0.230248 \\
\hline Left-hand finger & 0.098181 & 0.244009 & 0.02068 & 0.051400 & 0.40236 & 0.692430 \\
\hline Left thigh & -0.191410 & 0.191022 & -0.13032 & 0.130059 & -1.00203 & 0.330377 \\
\hline Left leg & 0.084039 & 0.163686 & 0.01921 & 0.037423 & 0.51341 & 0.614269 \\
\hline Left foot & 0.071433 & 0.264815 & 0.01378 & 0.051074 & 0.26975 & 0.790601 \\
\hline Right thigh & -0.061732 & 0.186596 & -0.04464 & 0.134918 & -0.33083 & 0.744812 \\
\hline Right leg & 0.180544 & 0.181837 & 0.04235 & 0.042656 & 0.99289 & 0.334688 \\
\hline Right foot & 0.122981 & 0.188374 & 0.02293 & 0.035117 & 0.65286 & 0.522581 \\
\hline
\end{tabular}

Li et al. suggested the feasibility of assessing skin involvement in SSc with skin ultrasound imaging. This study showed that echogenicity of skin correlated with skin thickness and the local mRSS. The results obtained by Li et al. [22] indicated that the ultrasound-measured skin thickness parameter was more sensitive than the mRSS in detecting skin involvement in SSc patients.

Other studies have suggested that US examination of only one anatomical site such as the proximal phalanx of the finger might reflect local skin involvement assessed by palpation and the overall skin involvement [16, 22-24].

According to Naredo et al. [24], US examination allows for a detailed image of the skin layers allowing for a reliable measurement of dermal thickness in SSc patients. These authors have suggested that ultra-high-frequency ultrasound was a tool that could provide a precise identification and measurement of dermal thickness. Moreover, they reported that dermal thickness in the finger is significantly higher in patients with SSc than the controls while in the forearm it was significantly lower in patients with SSc than the controls [24].
We argue that because mRSS may not be sensitive enough to detect slight but relevant changes in skin thickness over time [12], SWE should at least complement if not be a substitute for the mRSS.

\section{Conclusions}

In this study, the authors have shown that elastography is a useful method for the measurement of skin thickness in patients with SSc. The mean values obtained in the areas indicated in the results' description allow for the conclusion that these areas of the body are the most important for diagnosing and determining the severity of negative symptoms. Moreover, elastography is a reliable method of determining skin involvement in patients with systemic sclerosis and the results correlate positively to the RSS. That correlation was confirmed by the results indicating that both researchers showed a significant influence of elastography on the Rodnan test when examining the finger of the right hand. These results in the right-hand 
Table 4. The regression analysis of the results of elastography examination in different areas of the body in correlation to the results of the Rodnan skin score (researcher no. 2)

\begin{tabular}{|c|c|c|c|c|c|c|}
\hline \multirow[t]{2}{*}{$N=38$} & \multicolumn{6}{|c|}{$\begin{array}{c}\text { Rodnan 2 } \\
R=0.97622957 \\
R^{2}=0.95302417 \\
\text { Corrected } R^{2}=0.89775850 \\
F(20.17)=17.244, p<0.00001 \\
\text { Standard error of estimation: } 1.7615\end{array}$} \\
\hline & $b^{\star}$ & $\begin{array}{l}\text { Standard error } \\
\text { of } b^{\star}\end{array}$ & $b$ & $\begin{array}{l}\text { Standard error } \\
\text { of } b\end{array}$ & $t(17)$ & $p$ \\
\hline Constant & & & -8.50753 & 1.680552 & -5.06234 & 0.000096 \\
\hline Face & 0.111283 & 0.104130 & 0.05019 & 0.046960 & 1.06870 & 0.300147 \\
\hline Neck & -0.098964 & 0.133538 & -0.05945 & 0.080217 & -0.74109 & 0.468751 \\
\hline Anterior chest & 0.153806 & 0.139962 & 0.06525 & 0.059379 & 1.09891 & 0.287128 \\
\hline Abdomen & -0.130302 & 0.119819 & -0.08378 & 0.077042 & -1.08749 & 0.292001 \\
\hline Upper back & 0.143349 & 0.083408 & 0.12771 & 0.074309 & 1.71864 & 0.103835 \\
\hline Lower back & 0.036628 & 0.107149 & 0.01731 & 0.050631 & 0.34184 & 0.736661 \\
\hline Right upper arm & -0.017918 & 0.094170 & -0.00548 & 0.028803 & -0.19028 & 0.851347 \\
\hline Right forearm & 0.225021 & 0.114227 & 0.05980 & 0.030355 & 1.96994 & 0.065360 \\
\hline Right hand & -0.167625 & 0.154112 & -0.03607 & 0.033163 & -1.08768 & 0.291917 \\
\hline Right-hand finger & 0.440596 & 0.136012 & 0.07854 & 0.024247 & 3.23939 & 0.004821 \\
\hline Left upper arm & -0.058655 & 0.126804 & -0.02771 & 0.059915 & -0.46256 & 0.649544 \\
\hline Left forearm & -0.138056 & 0.172306 & -0.03890 & 0.048552 & -0.80122 & 0.434059 \\
\hline Left hand & 0.465691 & 0.163961 & 0.09707 & 0.034175 & 2.84026 & 0.011304 \\
\hline Left-hand finger & 0.142796 & 0.153460 & 0.03278 & 0.035229 & 0.93051 & 0.365132 \\
\hline Left thigh & 0.026484 & 0.120136 & 0.01965 & 0.089142 & 0.22045 & 0.828145 \\
\hline Left leg & 0.151725 & 0.102944 & 0.03780 & 0.025650 & 1.47386 & 0.158796 \\
\hline Left foot & -0.127396 & 0.166545 & -0.02678 & 0.035006 & -0.76493 & 0.454800 \\
\hline Right thigh & -0.098426 & 0.117352 & -0.07756 & 0.092472 & -0.83872 & 0.413264 \\
\hline Right leg & 0.133711 & 0.114359 & 0.03418 & 0.029236 & 1.16922 & 0.258450 \\
\hline Right foot & 0.139044 & 0.118470 & 0.02825 & 0.024069 & 1.17366 & 0.256714 \\
\hline
\end{tabular}

finger can be treated as an important diagnostic indicator and predictor for the severity of the negative symptoms associated with SSc. Both statistical analyses confirmed the reliability of the SWE examination method in patients with SSc and identified predictive body areas.

Elastography examination is also a dependable method of assessing the progression of SSc. High elastography scores correlate with a high RSS. In the authors' opinion, this study suggests that elastography is a reliable method to examine SSc progression and provides one useful site of examining allowing it to be an easy, cheap, and repeatable examination tool. The results of this study overlap and show the reliability of elastography as a method of assessing skin involvement. However, there is a need for an additional multicentre study to validate the results by independent physicians and check the compatibility of their outcomes. The use of US in the evaluation of the skin can allow for earlier diagnosis and better disease status monitoring. There is a need for the development of a standard operating procedure of examination for physicians treating patients with SSc. Despite the limitations of this study such as a small treatment group and only two specialists performing the research, to the best of our knowledge it is the first study to compare the cRSS with SWE and test the reliability of SWE as a method of skin assessment among SSc patients. The next step in the research process should be a multicentre research project with the participation of a larger group of specialists. The authors of this study believe that SWE skin assessment can be a sensitive testing method to detect skin involvement in SSc patients. The widespread availability of US machines could make this the preferred method used by clinicians as it is a non-invasive and relatively cheap method.

\section{Conflict of interest}

The authors declare no conflict of interest. 


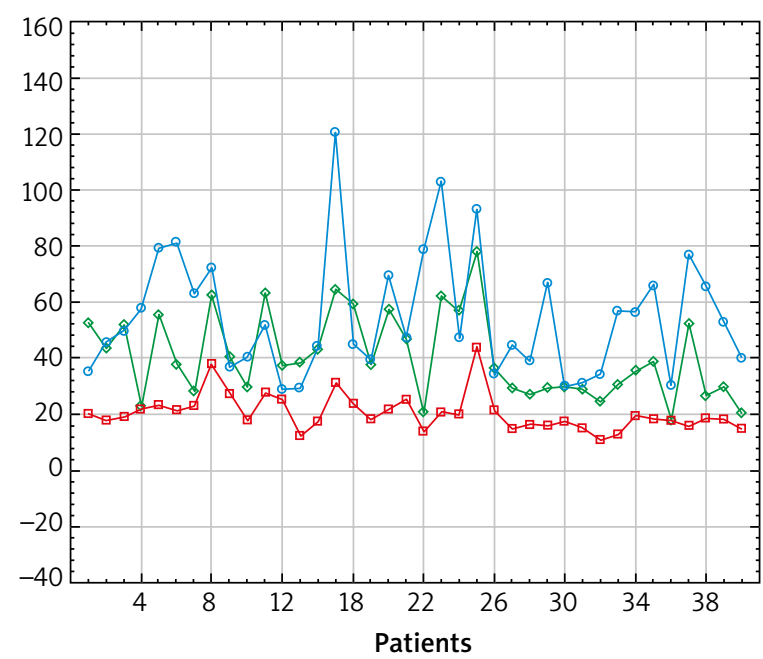

$\rightarrow$ Cluster $1 \rightarrow$ Cluster $2 \rightarrow$ Cluster 3

Figure 1. The results of cluster analysis using the k-mean elastography results in the studied areas of the body in the SSc group

\section{References}

1. LeRoy EC, Medsger TA, et al. Criteria for the classification of early systemic sclerosis. J Rheumatol 2001; 28: 1573-6.

2. Coentro JQ, Pugliese E, Hanley G, et al. Current and upcoming therapies to modulate skin scarring and fibrosis. Adv Drug Deliv Rev 2019; 146: 37-59.

3. Politikou O, Giesen T, Reissner L, et al. Hand and wrist joint procedures in patients with scleroderma: a systematic review. J Hand Surg Eur Vol 2019; 44: 402-7.

4. Hunzelmann N. Current treatment of systemic scleroderma. Hautarzt 2018; 69: 901-7.

5. Amjadi S, Maranian P, Furst DE, et al. Course of the modified Rodnan skin thickness score in systemic sclerosis clinical trials: analysis of three large multicenter, double-blind, randomized controlled trials. Arthritis Rheum 2009; 60: 2490-8.

6. Clements PJ, Lachenbruch PA, et al. Skin score: a semiquantitative measure of cutaneous involvement that improves prediction of prognosis in systemic sclerosis. Arthritis Rheum 1990; 33: 1256-63.

7. Clements PJ, Hurwitz EL, Wong WK, et al. Skin thickness score as a predictor and correlate of outcome in systemic sclerosis: high-dose versus low-dose penicillamine trial. Arthritis Rheum 2000; 45: 2445-54.

8. Wiese AB, Berrocal VJ, Furst DE, et al. Correlates and responsiveness to change of measures of skin and musculoskeletal disease in early diffuse systemic sclerosis. Arthritis Care Res 2014; 66: 1731-9.

9. Amjadi S, Maranian P, Furst DE, et al. Course of the modified Rodnan skin thickness score in systemic sclerosis clinical trials: analysis of three large multicenter, double-blind, randomized controlled trials. Arthritis Rheum 2009; 60: 2490-8.

10. Shand L, Lunt M, Nihtyanova S, et al. Relationship between change in skin score and disease outcome in diffuse cutaneous systemic sclerosis: application of a latent linear trajectory model. Arthritis Rheum 2007; 56: 2422-31.

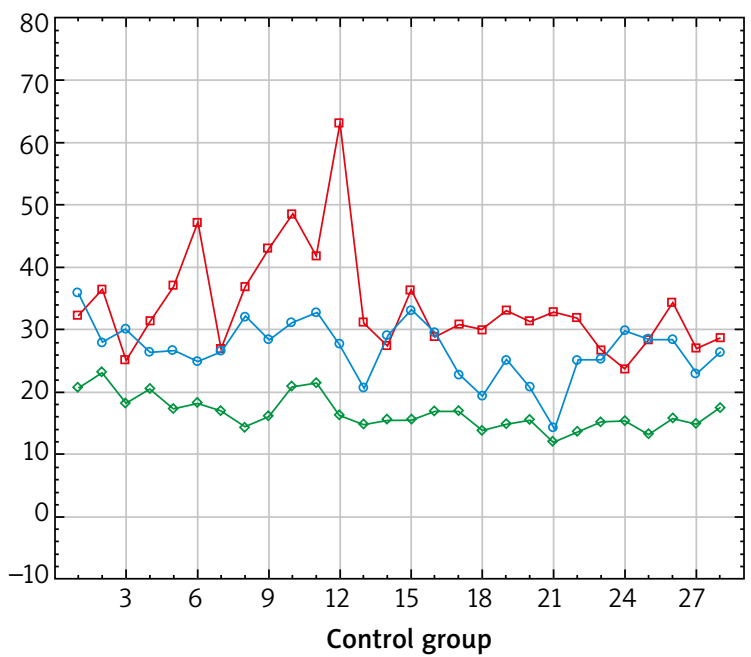

$\rightarrow$ Cluster $1 \rightarrow$ Cluster $2 \rightarrow$ Cluster 3

Figure 2. The results of cluster analysis using the k-mean elastography results in the examined body areas in the control group

11. Sedky MM, Fawzy SM, El Baki NA, et al. Systemic sclerosis: an ultrasonographic study of skin and subcutaneous tissue in relation to clinical findings. Skin Res Technol 2013; 19: 78-84.

12. Kang T, Abignano G, Lettieri G, et al. Skin imaging in systemic sclerosis. Eur J Rheumatol. Eur J Rheumatol 2014; 1: 111-6.

13. Sulli A, Ruaro B, Smith V, et al. Subclinical dermal involvement is detectable by high frequency ultrasound even in patients with limited cutaneous systemic sclerosis. Arthritis Res Ther 2017; 19: 61.

14. van den Hoogen F, Khanna D, Fransen J, et al. Classification criteria for systemic sclerosis: an American College of Rheumatology/European League against Rheumatism collaborative initiative. Arthritis Rheum 2013; 65: 2737-47.

15. Sobolewski P, Maślińska M, Zakrzewski J, et al. Applicability of shear wave elastography for the evaluation of skin strain in systemic sclerosis. Rheumatol Int 2020; 40: 737-45.

16. Sousa-Neves J, Cerqueira M, Santos-Faria D, et al. Ultrasound assessment of skin thickness performed on fingers: a tool for estimating overall severity of skin disease in systemic sclerosis patients? Acta Reumatol Port 2017; 42: 339-40.

17. Aryan A, Alaeen H, Dadgostar M, et al. Sonoelastography for skin evaluation in sclerodermic patients. Int J Prev Med 2019; 10: 91.

18. Liu H, Hou Y, Zhu QL, et al. A preliminary study of skin ultrasound in diffuse cutaneous systemic sclerosis: does skin echogenicity matter? PLoS One 2017; 12: e0174481.

19. Dźwigała M, Sobolewski P, Maślińska M, et al. High-resolution ultrasound imaging of skin involvement in systemic sclerosis: a systematic review. Rheumatol Int 2021; 41: 285-95.

20. Yang Y, Yan F, Wang L, et al. Quantification of skin stiffness in patients with systemic sclerosis using real-time shear wave elastography: a preliminary study. Clin Exp Rheumatol 2018; 113: 118-25.

21. Yang Y, Qiu L, Wang L, et al. Quantitative assessment of skin stiffness using ultrasound shear wave elastography in systemic sclerosis. Ultrasound Med Biol 2019; 45: 902-12. 
22. Li H, Furst DE, Jin $\mathrm{H}$, et al. High-frequency ultrasound of the skin in systemic sclerosis: an exploratory study to examine correlation with disease activity and to define the minimally detectable difference. Arthritis Res Ther 2018; 20: 181.

23. Santiago T, Alcacer-Pitarch B, Salvador MJ, et al. A preliminary study using virtual touch imaging and quantification for the assessment of skin stiffness in systemic sclerosis. Clin Exp Rheumatol 2016; 100: 137-41.

24. Naredo E, Pascau J, Damjanov N, et al. Performance of ultrahigh-frequency ultrasound in the evaluation of skin involvement in systemic sclerosis: a preliminary report. Rheumatology 2020; 59: 1671-78. 